(C) 1973 IEEE. Personal use of this material is permitted. However, permission to reprint/republish this material for advertising or promotional purposes or for creating new collective works for resale or redistribution to servers or lists, or to reuse any copyrighted component of this work in other works must be obtained from the IEEE.

\title{
A LONGITUDINAL STABILITY CRITERION FOR BUNCHED BEAMS
}

F.J. Sacherer
CERN, Geneva, Switzerland

\section{$\underline{\text { Summary }}$}

The unstable motion of a bunched beam consists of rigid-bunch (dipole) and higher bunch-shape oscillations of the individual bunches (individual-bunch modes), plus perhaps coupled motion of the different bunches (coupled-bunch modes). Stability is achieved either by decoupling the bunches or by a spread in synchrotron frequencies within a bunch. A stability criterion analogous to the Keil-Schnell criterion for coasting beams is given which includes the effect of a beam interacting with perfectly conducting walls, resistive walls, and resonant structures. Some examples for the CERN accelerators are included.

\section{Introduction}

Most previous work has concentrated on rigid-bunch motion, driven by resonant elements in the vacuum chamber, cavities, or the wall resistance. ${ }^{2-9}$ However, the higher modes can also be excited. ${ }^{10-12}$ For example, a resonator of sufficiently high frequency that one or more oscillations occur during the passage of a bunch will excite primarily the higher modes. Also, the space-charge force depends on the variation of the 1 ine density within a bunch, and therefore increases with mode number. On the other hand, the resistive-wall wake decays relatively little over one bunch and is insensitive to the density variations of the higher modes; it drives mostly the rigid-bunch mode, as in the transverse case.

This paper presents a general and hopefully easy to use stability criterion for the higher modes of oscillation as well as the rigid-bunch mode. Four ingredients are necessary:

i) oscillation modes

ii) coherent frequency shifts $\Delta$ us

iii) decoupling criterion

iv) stability critcrion.

The derivations are given in another paper, ${ }^{13}$ and only the results are presented here.

\section{Classification of modes}

For bunched beams, the dominate force is the external synchrotron force, and the particle motion is approximately circular in the nomalized $z-\dot{z} / \omega_{\mathrm{s}}$ phase plane (Fig. 1). An exactly circular distribution $g_{0}(r)$ is stationary, while small oscillations about the stationary distribution have the form

$$
g(r, \neq, t)-R_{m}(r) e^{-i m \phi} e^{-i \omega t}
$$

and oscillate with the frequency

$$
\omega=m \omega_{s}+\Delta \omega_{m},
$$

where is is the synchrotron frequency, and $m=1$ for dipole modes, $m=2$ for quadrupole modes, etc. If Eq. (1) is inserted into the 1 inearized vlasov equation, an integral equation results for the radial mode patterns $R_{\mathrm{Tn}}(r)$ and the coherent frequency shifts $\mathrm{w}_{\mathrm{m}}$. For the case; considered here, and probably in general, the solutions have the form

$$
R_{m}(r) \approx r^{m-1} \frac{d g_{0}}{d r}
$$

provided frequency spreads are neglected. A few of the low-order oscillation modes are sketched in Fig. 1.
In addition, coupled motion of the different bunches occurs if their oscillation frequencies are nearly equal. For $M$ identical bunches, there are $M$ coupledbunch modes of oscillation. These are designated by the index $n$, which specifies the phase difference $2 \pi n / M$ between adjacent bunches.

Notice that two indices are necessary to describe the complete oscillation: m specifies the type of oscillation the individual bunches are undergoing, while $n$ describes how these individual-bunch modes are linked together in the larger coupled-bunch pattern. This convention for the indices $m$ and $n$ will be observed in the following.

Given the shape of the modes and the beam-equipment coupling impedance $Z_{\|}(\omega)$, a straightforward procedure exists for computing $\Delta \omega_{m} \cdot{ }^{14}$ Results for perfectly conducting walls, resistive walls, and resonators are given in the next section.

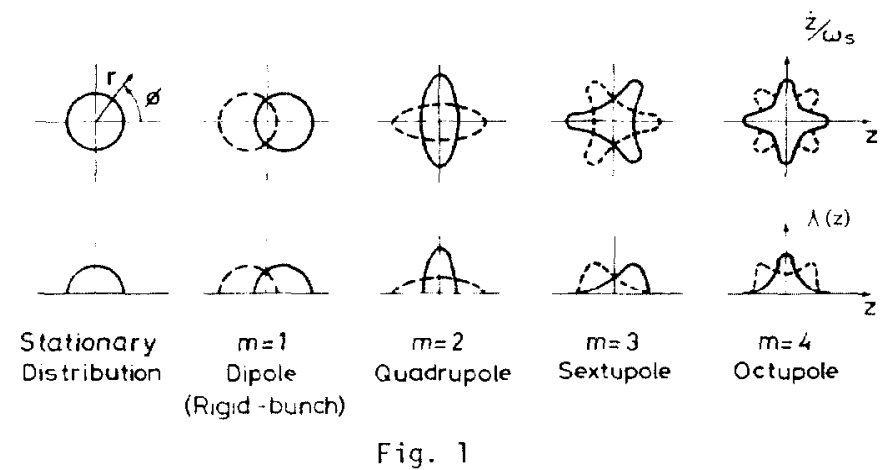

Growth-rates in the absence of frequency spreads

The growth-rate is

$$
\frac{1}{\tau}=\operatorname{Im} a_{\mathrm{m}},
$$

and the motion is unstable if $\operatorname{Im} c \omega_{m}$ is positive. It is ronvenient to write the expressions for the frequency Sifts in the form

$$
\frac{A \omega_{m}}{\omega_{S}}=\frac{Z I}{v \cos \omega_{s}} \times \text { form factors }
$$

where $z$ is a characteristic impedance for the inter action in question; $V$ is the peak accelerating voltage per turn; $\phi_{s}$ is the synchronous phase, with the convention that $\mathrm{V} \cos \phi_{\mathrm{S}}$ is positive below transition and negative above.

\section{Perfectly conducting walls}

For a bunch with approximately parabolic line density,

$$
A_{1} m=\sqrt{m} A_{s c} \text {, }
$$

where

$$
\begin{aligned}
& \frac{i \omega s c}{\omega_{s}}=0.152 \frac{z_{s c} / \mathrm{k} / \mathrm{I}}{\mathrm{v} \cos \phi_{\mathrm{s}}} \frac{\mathrm{m}^{2}}{\mathrm{hB}^{3}}, \\
& \left|z_{s c} / k\right|=\frac{g_{c}}{2 B y^{2}} Z_{0},
\end{aligned}
$$


and

$g_{c}=1+2 \ln$ (vacuum chamber radius/beam radius).

$Z_{s c}$ is the usual lorgitudinal coupling impedance for mode $\mathrm{k} ; Z_{0}=377 \mathrm{ohm}$; I is the total current in $M$ bunches; $h$ is the $\mathrm{RF}$ hamonic number (usually equal to M); $B$ is the buncing factor (ounch length/bunch separation).

Note that the frequency shift $L \omega_{m}$ is real, depends strongly on the bunching factor, and increases with the square root of the tode number $m$. (The analogous freciency shift for coasting beams increases linearly with node number.) The square-root dependence on mode number is characteristic for bunched beams, and is apparently Sue to the $\mathrm{r}^{\text {m }}$ factor in the radial distribution (3), which constrains the motion more and more to the bean edge.

\section{Resistive walls}

The effect of a smooth round vacuun chamber on the dipole mode is

$$
\frac{\Delta \omega_{1}}{\omega_{S}}=0.0134 \frac{\left|z_{s k i n}\right| I}{V \cos \phi_{S}} \frac{M^{3 / 2}}{h} G\left(\frac{Q_{S}+n}{M}\right)
$$

where

$$
\left|z_{\text {skin }}\right|=\frac{1}{\sqrt{2}} \beta \frac{b}{b} z_{c},
$$

and $c / b$ is the ratio of skin depth at the revolution frequency $\omega_{0}$ to the vacuum chamber radius; $Q_{S}$ is the number of synchrotron cscillations per revolution, $Q_{\mathrm{S}}=\omega_{\mathrm{S}} / \omega_{0} ;$ anc $\mathrm{G}$ is a bunch function analogous to the one defined by Courant and Sessler for transverse motion: ${ }^{15}$

$$
G(Q)=\sum_{s=1}^{\infty} \frac{e^{2 \pi i s Q}}{s^{5 / 2}}
$$

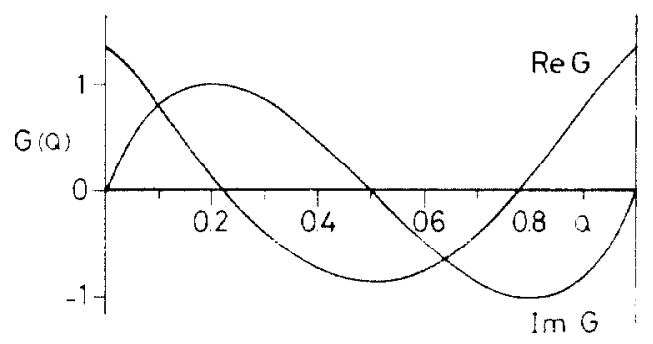

Fig. 2

Its maximur real or imaginary part is about unity, and Im $G$ is positive if its argument is less than one-half (Eig. 2). Thus for single-bunch motion $(M=1, n=0$, and $I \rightarrow I / M)$, the bunches are unstable below transition and stable above, assuming that $Q_{3}<1 / 2$. For coupled motion, about half the modes are unstable in all cases. Robinson ${ }^{3}$ has found a similar result, except with $\mathrm{s}^{3 / 2}$ instead of $\mathrm{s}^{5 / 2}$ in $\mathrm{Eq}$. (9). I think Eq. (9) is correct, but in any case the difference is negligible.

The frequency shifts for the higher modes are estimated to be about

$$
L_{3} m_{m} \cdot B^{m} \Delta b_{1}
$$

where $B$ is the bunching factor.

\section{Resonator}

We assume a cavity or resonant element characterized by a shunt or parallel resistance $R_{S}$, resonant frequency $f_{r e s}$ (or radian frequency $\omega_{r e s}$ ), and quality factor $Q$. Transit time factors are ignored. Then

$$
\frac{\Delta w_{m}}{w_{s}}=0.159 \frac{R_{s} I}{V \cos \phi_{s}} \frac{M}{B h} D F_{m}(\Delta \phi) \text {. }
$$

$F_{\mathrm{m}}$ is a form factor that specifies the efficiency with which the resonator can drive a given mode. It depends on the phase change $\Delta \phi$ that occurs during the passage of a bunch (see Fig. 3):

$$
\Delta \phi=2 \pi f_{\text {res }} x \text { bunch length in seconds. }
$$

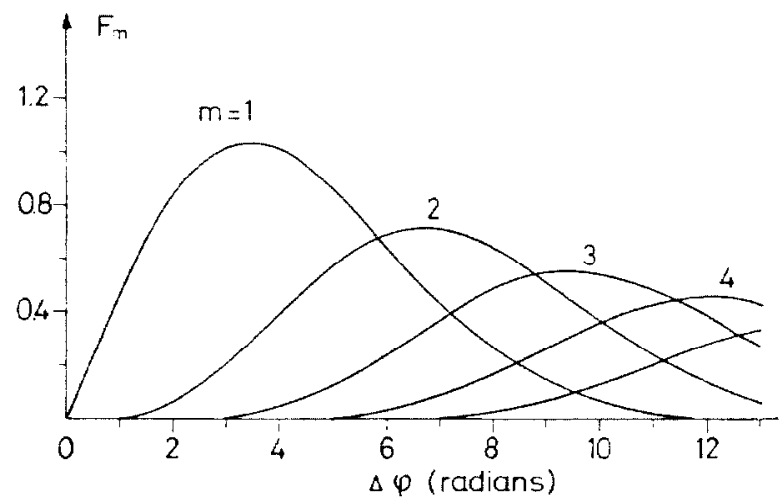

Fig. 3

'the maximum value of $\mathrm{F}_{1}$ for the dipole mode occurs when $\Delta \phi \simeq \pi$ so that an approximately linear waveform acts on the bunch. Similarly, the quadrupole or breathing mode is most efficiently driven when $\Delta \vec{\psi}$ is near $2 \pi$, and so on for the higher modes. In general, mode $m$ is most efficiently driven when the resonator frequency is $\mathrm{f}_{\text {res }}=\mathrm{m} \mathrm{f}_{\text {crit }}$, where

$$
f_{\text {crit }}=\frac{M}{2 B} f_{0}
$$

is the most efficient frequency for driving dipole modes ( $f_{0}$ is the revolution frequency in $\mathrm{Hz}$ ). For these frequencies, the maximum value of $F_{m}$ is approximately $1 / \sqrt{\mathrm{m}}$.

The factor $\mathrm{D}$ in $\mathrm{Eq}$. (10) depends on the attenuaLiun $e^{-\alpha}$ of the incuced signal vetween bunches, where

$$
\begin{aligned}
\alpha & =2 \pi \text { if } \times \text { time between bunch centres } \\
& =\frac{\Delta \phi}{2 Q B}
\end{aligned}
$$

and if $=f_{\text {res }} / 2 Q$ is the bandwidth of the resonator.

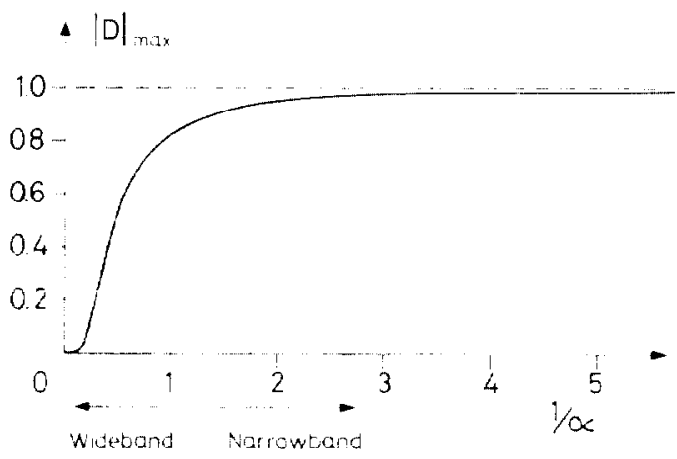

Fig. 4 
The maximum value of $|D|$ is about unity for narrow-band resonators, with little attenuation between bunches, and approaches zero for wide-band resonators (Fig. 4).

Narrow bana, a $\ll I$ :

$$
D \simeq \frac{\delta f}{f_{\text {res }}-\text { integer } \times f_{0}-i \delta f}
$$

and the coupled-bunch mode $\mathrm{n}$ is excited when

$$
\mathrm{f}_{\text {res }} \simeq \text { integer } \times \mathrm{Mf}_{0} \pm \mathrm{nf}_{0} .
$$

wilie band, $\alpha \gg 1$ :

$$
D \simeq-2 \alpha e^{-\alpha} e^{2 \pi i n / M} \sin \left(2 \pi f_{\mathrm{res}} / \mathrm{Mf}_{0}\right),
$$

and coupled-bunch modes near $n= \pm M / 4$ (phase difference between bunches of $t \pi / 2$ ) are most strongly excited.

Geverat case, any a:

$$
D=i \alpha\left[\frac{1}{1-e^{x_{+}}}-\frac{1}{1-e^{x_{-}}}\right] \text {, }
$$

where

$$
\mathrm{x}_{ \pm}=\frac{2 \pi i}{\mathrm{M}}\left(\mathrm{n} \pm \frac{\mathrm{f}_{\mathrm{res}}}{\mathrm{f}_{0}}\right)-\alpha .
$$

Examples are shown in Fig. 5.

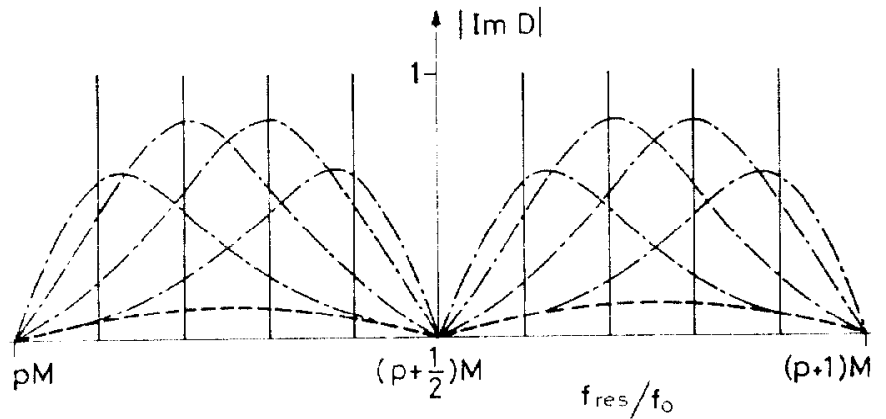

$P=$ arbitrary integer

- - - - wide-bani resonance with attenuation $e^{-4}$ between bunches

-. - - - intermediate case with attenuation $e^{-1}$ between bunches narrow-bund resonance witin at atenuation between bunches

Fig. 5 'im $D$ versus frequency for the hypothetical case of 10 bunches $(M=10)$

In all cases, modes $n=0$ and $n=M / 2$ (if $M$ is cven) are not excited. Also, if the bunches are decoupled from one another, the motion is stable because $\mathrm{n}=$ Dor a single bunch. (Actually, the snall phase chane per revolution due to the synchrotron oscillarion results in a weak instability for mode $n=0$, but this is nerrigible compared with the other modes and has been neglected here.)

\section{Decoupling criterion}

The basic mechanism for decoupling the bunches is a sprcad in the Erequercies of the indivitual hunches. A rule-of-thumb for decoupling is that the r.m.s. spread in individual bunch frequencies should exceed the frequency silife tion cue to the coupling force: ${ }^{i 6}$

spread > shift.
If a beam control system is acting, somewhat larger spreads are required. ${ }^{8}$ The spread may be induced externally by modulating the RF voltage, or may arise naturally from a difference $\Delta N$ in bunch populations. In the last case, a convenient criterion for decoupling is

$$
\ln \Delta \omega_{S c}\left|\left(\frac{\Delta N}{N}\right)_{\text {rms }}>\right| \Delta w_{m}^{\prime} \mid
$$

where the prime indicates that the space-charge shift (6) stould be omitted (it does not contribute to the coupling). Since $(\Delta \mathrm{N} / \mathrm{N})_{\mathrm{rms}}$ is usually less than $5 \%$ (or a full spread of 20\%), large space-charge shifts are required for decoupling.

\section{Stability criterion}

If within-bunch frequency spreads are taken into account, the following dispersion relation can be derived for the different modes:

$$
1=\frac{\Delta \omega_{m}}{w_{m}} \int_{0}^{\infty} \frac{r^{2 m}}{\omega-m \omega_{s}(r)} \frac{d g_{0}}{d r} d r,
$$

where

$$
W_{m}=\int_{0}^{\infty} r^{2 m} \frac{d g_{0}}{d r} d r,
$$

and $\Delta w_{m}$ is the sum from all interactions, space charge, resistive wall, resonators, etc. We define $S$ as the spread in $\omega_{s}$ between centre and edge of the bunch (full spread) due to the non-linearity of the synchrotron force; it is plutted in Fig. 6 as a function of the bunching factor and the parameter $l^{\prime}=\sin \phi_{\mathrm{S}}$.

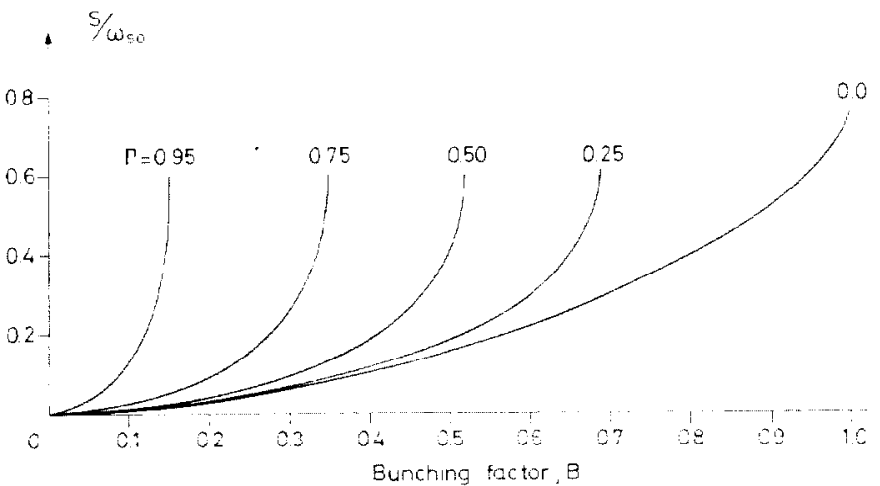

Fig. 6

Equation (16) specifies the stable regions in the complex Lim/S plane, and these are plotted in Fig. 7 for the smooth distribution

$$
g(x)+\left(1-r^{2}\right)^{2}
$$

with zero slope at the beam edge. Following the example of Keil and Schnell 1 for coasting beans, we can approximate the stability boundary by semicirclus to sive

$$
\mathrm{S} \cdot \frac{4}{\sqrt{\mathrm{m}}} \cdot u_{\mathrm{m}} \mid
$$

for stability. This is analogous to the cuating-beart criterion

$$
\text { (full bpread at base) } \div \frac{4}{k} L_{k}
$$

for mode $k$. The criterion ( 18 ) has been derived previously ${ }^{17}$ for the rigid-bunch mode $(m=1)$. 
For space charge, $\Delta \omega_{m}$ increases as $r$ and therefore the threshold is the same for all modes, just as for coasting beams. For a resonator, $\Delta u_{m}$ decreases as $1 / \sqrt{m}$ and therefore the higher modes require less frequency spread. Stated differently, m times more shunt resistance is required to excite mode m at its critical Erequency $\mathrm{mf}_{\mathrm{c}}$ it than is necessary to excite the dipole mode at its critical frequency. However, once the threshold is exceeded, either by the action of space charge or the resonator itself, the growth rates for the higher modes decrease only as $1 / \sqrt{m}$.

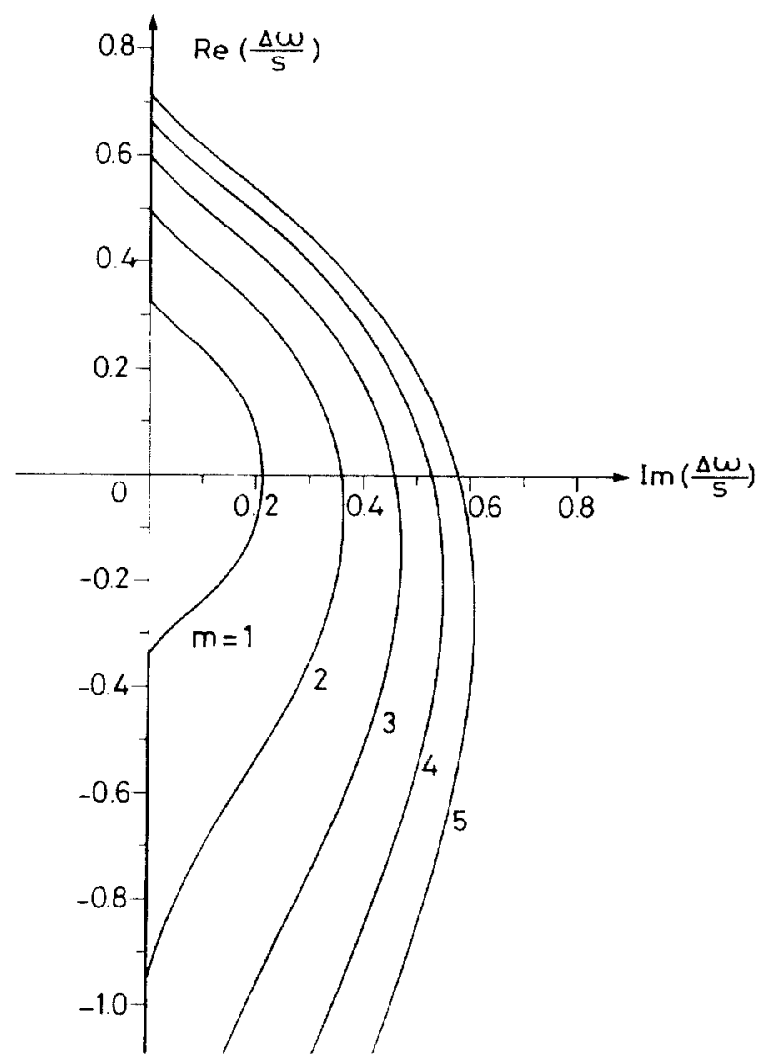

Fig. 7 Stability diagram. Regions to the left of the contour are stable for the indicated mode, regions to the right are unstable.

\section{Examples}

Some examples for the CERN machines are given in Table 1, including resonator shunt impedances necessary to drive dipole modes, namely

$$
\begin{aligned}
& \mathrm{R}_{\text {co }} \text { to reach threshold } \\
& \mathrm{R}_{50} \text { for } 50 \mathrm{msec} \text { growth time } \\
& \mathrm{R}_{10} \text { for } 10 \mathrm{msec} \text { growth time. }
\end{aligned}
$$

These are computed assuming that the quality factor is larger than $1 /$ (bunching factor) and that $\mathbf{f}_{\text {res }} \approx \mathbf{f}_{\text {crit }}$, so that the form factors $D$ and $F$ in Eq. (10) are about unity. $R_{\infty}$ is determined by

$$
\left|A_{\mathrm{SC}}\right|+A_{n} \text { due to resonator } \mid=\frac{1}{4} \mathrm{~S} \text {, }
$$

and $R_{50}$ by

$R_{50}=R_{\infty}+5 R$ required to give $\left|\Delta \omega_{1}\right|=20 \mathrm{rad} / \mathrm{sec}$, and similarly for $R_{10}$. The resistive-wall growth rates are negligible $\left(z_{\text {skin }}{ }^{n} 1\right.$ to 10 ), and are not included.
PSB (Booster): Because of the large space-charge force, all modes are well outside the stable region during most of the acceleration cycle. Also, only small shunt resistances are required to drive dipole modes, $70 \Omega$ for $50 \mathrm{msec}$ e-folding time and $350 \Omega$ for $10 \mathrm{msec}$. On the other hand, it is unlikely that elements exist in the machine with such small resonant frequencies. One expects frequencies of $30 \mathrm{MHz}$ and above, which will drive the dipole modes with reduced efficiency, or drive higher modes. To drive mode $m=4$ with an e-folding time of $10 \mathrm{msec}$ requires $\sqrt{\mathrm{m}} \times 350=700 \Omega$. A1so the revolution frequency changes by a factor of $2 \frac{1}{2}$ during the cycle so that many resonance regions $\mathrm{f}_{\text {res }} / \mathrm{f}_{0} \approx$ integer are swept through.

In fact, dipole and higher bunch-shape oscillations occur, but it is too early to decide if they are due to bugs in the RF system or to beam-equipment interactions.

YS (1970): We concentrate on the region after transition when the revolution frequency is approximately constant. With a constant accelerating voltage of $115 \mathrm{kV}$, the RF bucket is large compared with the bunch, and the frequency spreads are insufficient to maintain stability. A strong dipole instability with growth time of $10 \mathrm{msec}$ was observed when electrostatic septum tanks (2) were installed. One was measured (by H.H. Umstätter) and found to have $R_{S}=18 \mathrm{k} \Omega, f_{\text {res }} \simeq 60$ to $90 \mathrm{MHz}$ depending on the position of the septa, and $Q \simeq 700$. This was cured by means of damping resistors, but a slower dipole instability remained with growth times of about $50 \mathrm{msec}$. This is probably due to a parasitic resonance in the $14 \mathrm{RF}$ cavities with $\mathrm{R}_{\mathrm{S}}=14 \times 800=11.2 \mathrm{k} \Omega$, f fes spread between 46 and $51 \mathrm{MHz}$, and $Q \simeq 20$. The computed $e^{-}$ folding time of $28 \mathrm{msec}$ using Eq. (10) and assuming a single resonant frequency of $48 \mathrm{MHz}$ is in reasonable agreement with observation. More exact computations using the measured impedance curves and including the effect of the beam control system are reported in Refs. 6,8 , and 9. The present cure is to reduce the size of the bucket by voltage reduction.

PS (1972): The threshold impedance increases from zero to $10 \mathrm{k} \Omega$ when the vol tage is reduced until $85 \%$ of the bucket is filled. At present, no instabilities are observed.

Future FS: At $10^{13}$ particles, voltage reduction may not be sufficient, even allowing for a $50 \%$ increase in longitudinal emittance. A feedback system is being considered. ${ }^{9}$

ISR: At present, the bucket is very large compared with the bunch, so frequency spreads are small and space charge is sufficient to move all modes outside the stable region. A relatively large impedance of $15 \mathrm{k} \Omega$ is required for a $50 \mathrm{msec}$ growth time, but when this is divided by the coasting-beam mode number $k=f_{\text {res }} / f_{0}=97$, we $f$ ind

$$
\mathrm{R}_{50} / \mathrm{k}=155 \Omega \text {, }
$$

which is in the range of possible impedances. This ratio is even snaller for the higher modes since $R_{S}$ scales as $\sqrt{m}$ while $k$ scales as $\mathrm{m}$ :

$$
\mathrm{R}_{50} / \mathrm{k}=155 \mathrm{a} / \mathrm{m} \text {. }
$$

In fact, higher bunch-shape oscillations are observed and lead to a doubling of the longitudinal emittance. voltage reduction should cure this.

SPS: $\Lambda$ threshold impedance of about $1 \mathrm{M}$ is required for instability. 
Table 1: Computed parameters for dipole modes of instability

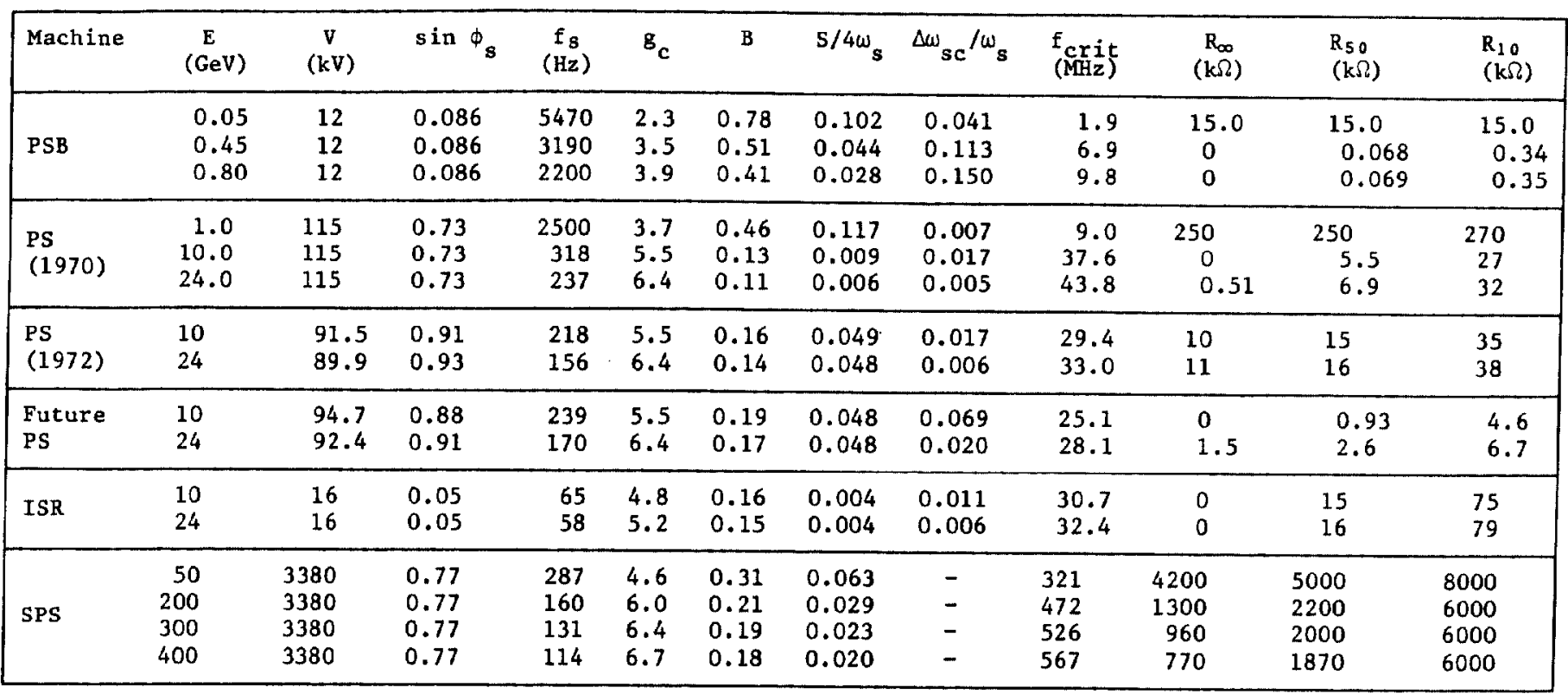

Table 2: Fixed parameters used for computing Table 1

\begin{tabular}{|lcccc|}
\hline Machine & $\begin{array}{c}\text { No. of particles } \\
\times 10^{12}\end{array}$ & $\begin{array}{c}\text { Bunch } \\
\text { eV.sec }\end{array}$ & $\begin{array}{c}\text { area } \\
\text { mrad }\end{array}$ & $\begin{array}{c}\text { B } \\
\text { Tesla/sec }\end{array}$ \\
\hline PSB & 2.5 per ring & 0.156 & 10 & 0.80 \\
PS & 1.5 & 0.156 & 10 & 1.9 \\
Future PS & 10.0 & 0.234 & 15 & 1.9 \\
ISR & 1.5 & 0.156 & 10 & 0.01 \\
SPS & 10.0 & 0.100 & 130 & 0.75 \\
\hline
\end{tabular}

\section{Acknowledgements}

I have profited very much from a close collaboration with D. Möhl. In particular, he read the manuscript and pointed out several improvements. Discussions with J. Gareyte and Y. Baconnier were also very helpful.

\section{References}

1. E. Keil and $W$. Schnell, Concerning longitudinal stability in the ISR, CERN-ISR-TH-RF/69-48 (1969).

2. V.L. Auslender, M.M. Karliner, A.A. Naumov, S.G. Yopov, A.N. Skrinskii and I.A. Shekhtman, Int. Conf. on High-Energy Accelerators, Frascati, Italy (1965), p. 339.

3. K.W. Robinson, SLAC Storage Ring Summer Study, 1965, Report SLAC-49, p. 69.

4. K.W. Robinson, Stability of beam in radiofrequency system, Cambridge report CE AL-1010 (1964).
5. C. Pellegrini, Linear theory of RF cavity, bunched-beam interaction, unpublished manuscript (1967).

6. Y. Baconnier, J. Gareyte and P. Lefèvre, National Particle Accelerator Conf. Chicago, 1971 [IEEE Trans. Nuclear Sci. Vol. NS-18, 1027 (1971)].

7. M.Q. Barton and E.C. Raka, see Ref. 6, p. 1032.

8. D. Boussard, J. Gareyte and D. Möhl, see Ref. 6, p. 1073.

9. D. Boussard and J. Gareyte, 8th Int. Conf. on HighEnergy Accelerators, CERN, 1971 (CERN, Geneva, 1971), p. 317 .

10. A.N. Lebedev, 6th Int. Conf. on High-Energy Accelerators, Cambridge, USA, 1967, p. 284.

11. Y. Baconnier, Bunch-shape instability induced by a cavity, CERN/MPS/DL, 70-15 (1970).

12. I. Gumowski, 8th Int. Conf. on High-Energy Accelerators, CERN, 1971 (CERN, Geneva, 1971), p. 360.

13. F.J. Sacherer, A longitudinal stability criterion for bunched beams, CERN/MPS/BR, 73-1 (1973).

14. F.J. Sacherer, Methods for computing bunched-beam instabilities, CERN/SI-BR/72-5 (1972).

15. E.D. Courant and A.M. Sessler, Rev. Sci. Instrum. 37, 1579 (1966).

16. F.J. Sacherer, Spring Study on Acceleratory Theory, 1972, Report to the $300 \mathrm{GeV}$ Advisory Machine Committee, CERN, p. 159.

17. H. Hübner and V.G. Vaccaro, Dispersion relations and stability of coasting beams, CERN-ISR-TH/70-44 (1970). 